\title{
Ethnic Specific Differences in Cholesterol Trafficking Following High Fructose Feeding
}

\author{
S. V. Harding and L. M. Goff \\ Diabetes and Nutritional Sciences Division, School of Medicine, King's College London, London, United Kingdom, \\ SE1 9 NH
}

This study aimed to assess ethnic-specific impacts of fructose on cholesterol metabolism of black West-African (BA) and white European (WE) men. Fifteen healthy men (7 BA/8 WE) undertook 24-hrs controlled feeding (25\% calories from fructose). Plasma cholesterol (TC), HDL, non-HDL, triglycerides (TG), red blood cell cholesterol (Chol), lathosterol (LS), campesterol (CS) and $\beta$-sitosterol (BS) concentrations were measured at baseline and $24 \mathrm{hrs}$ and changes assessed in each ethnicity by student and paired t-tests.

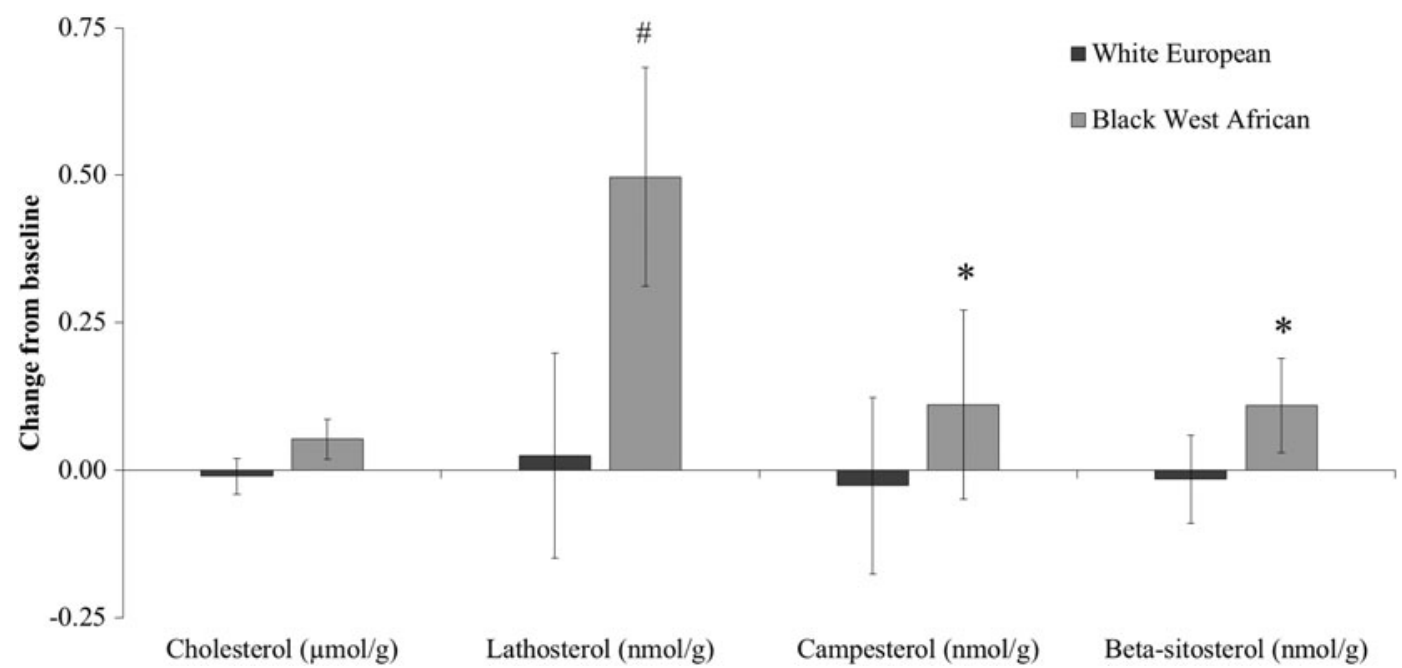

Fig. 1. Red blood cell sterol concentration changes at 24 hours folloeing high fructose feeding. Values are mean \pm SEM. Significant differences from baseline within ethnicity are denoted by $*$. ${ }^{*}$ Changes in lathosterol concentrations tended to be higher $(p=0.100)$ in Black West African men only, suggesting an increased cholesterol

Baseline TC $(5.13 \pm 0.68$ vs. $4.33 \pm 0.58, p=0.03)$ and non-HDL $(3.75 \pm 0.61$ vs. $3.07 \pm 0.51, p=0.03)$ were higher in BA but there were no differences in HDL or TG. There were no significant lipid changes $(\mathrm{TC}+0.21 \mathrm{mmol} / \mathrm{L}$; non-HDL $+0 \cdot 18 \mathrm{mmol} / \mathrm{L}$; HDL $+0.03 \mathrm{mmol} / \mathrm{L}$; TG $+0.05 \mathrm{mmol} / \mathrm{L})$ following fructose feeding in WE while BA TC $(+0.38 \mathrm{mmol} / \mathrm{L}, p=0.002)$, non-HDL $(+0.30$ $\mathrm{mmol} / \mathrm{L}, p=0.004)$ and HDL $(+0.08 \mathrm{mmol} / \mathrm{L}, p=0.02)$ increased. Baseline RBC Chol, LS, CS and BS were higher in BA compared to WE. The RBC Chol concentration following fructose was not different between ethnicities but did remain higher in BA (0.17 vs. $0.05 \mu \mathrm{mol} / \mathrm{g} \mathrm{RBC}, p=0.003)$. Changes between baseline and 24-hr for RBC Chol, LS, CS and BS are shown in Figure 1. The LS:Chol (mmol:mol chol) (5.6 vs. $4.2, p=0.06)$ tended to be higher in BA suggesting a higher cholesterol synthesis. Finally, CS (0.54 vs. 0.08 $\mathrm{nmol} / \mathrm{g} \mathrm{RBC}, p=0.02)$ and $\mathrm{BS}(0.34 \mathrm{vs} .0 .06 \mathrm{nmol} / \mathrm{g} \mathrm{RBC}, p=0.008)$ concentrations were higher and the BS:Chol $(1.9 \mathrm{vs} .1 .2, p=$ $0 \cdot 11)$ tended to be higher at $24 \mathrm{hrs}$ in BA, indicating higher cholesterol absorption. These data illustrate both ethnic specific differences in markers of cholesterol metabolism and a differential effect of fructose on these outcomes. 